\title{
O dvjema metodama integriranja nekih iracionalnih funkcija
}

\author{
Denis Benčec, Bojan Kovačić
}

\begin{abstract}
Sažetak
U nastavi matematičkih predmeta na veleučilištima, samostalnim visokim školama i fakultetima obrađuju se i integrali iracionalnih funkcija. Neki od tih integrala mogu se odrediti i pomoću trigonometrijske i pomoću hiperbolne zamjene, pri čemu se obično preferira prva zamjena. U članku se na dvama primjerima uspoređuju navedene metode, te se iznose njihove osnovne prednosti i nedostaci.
\end{abstract}

Ključni pojmovi: integriranje iracionalnih funkcija, trigonometrijska zamjena, hiperbolna zamjena

\section{Uvod}

U nastavi matematičkih predmeta na našim veleučilištima, samostalnim visokim školama i fakultetima, a posebno na onima koji izvode tehničke sveučilišne i stručne studije, obrađuju se osnove integralnoga računa. Među ostalima, obrađuju se neki posebni tipovi neodređenoga integrala iracionalnih funkcija. Termin posebni tipovi navodi se zato jer iracionalna funkcija općenito ne mora biti elementarno integrabilna. Spomenuti tipovi integrala najčešce se određuju metodom zamjene (supstitucije). Pritom se u nekim slučajevima mogu koristiti dvije vrste zamjene: trigonometrijska zamjena i hiperbolna zamjena. Na nastavi se obično odabere jedna vrsta zamjene koja se iskoristi za rješavanje zadatka, pa se potom zaključno napomene da se drugom zamjenom dobije jednak rezultat. Naše iskustvo pokazuje da studenti imaju značajnih poteškoća upravo s dokazivanjem jednakosti dobivenih rezultata. Te poteškoće najvećim dijelom proizlaze iz potrebe sređivanja i pojednostavljivanja različitih trigonometrijskih, odnosno hiperbolnih izraza. Zbog 
toga ćemo u ovom članku na konkretnim primjerima ukazati na navedene poteškoće, te usporediti načine rješavanja objema metodama navodeći osnovne prednosti i nedostatke svake metode.

\subsection{Pregled nekih definicija, funkcija i identiteta}

Radi nešto manje korištenoga termina standardna antiderivacija, podsjetimo na definiciju toga pojma (vidjeti npr. 2]).

Definicija 1. Neka je f integrabilna realna funkcija. Primitivnu funkciju funkcije f čiji je slobodni član (sumand koji ne sadrži nezavisnu varijablu) jednak nuli nazivamo standardna antiderivacija funkcije $f$.

Napomenimo da se u zadacima obično traži određivanje neodređenoga integrala (skupa svih primitivnih funkcija). Radi jednostavnosti, u odabranim primjerima ćemo odredivati samo standardnu antiderivaciju.

$\mathrm{U}$ odnosu na trigonometrijske i arkus funkcije, hiperbolne i area funkcije se rjede koriste. Zbog toga navodimo pravila pridruživanja i prirodne domene onih hiperbolnih i area funkcija koje ćemo koristiti u primjerima. U daljnjem tekstu pod pojmom pravilo podrazumijevamo pravilo pridruživanja kojim je zadana neka funkcija.

\begin{tabular}{|c|c|c|c|}
\hline Naziv & Oznaka & Domena & Pravilo \\
\hline sinus hiperbolni & sh & $\mathbb{R}$ & $\frac{e^{x}-e^{-x}}{2}$ \\
\hline kosinus hiperbolni & ch & $\mathbb{R}$ & $\frac{e^{x}+e^{-x}}{2}$ \\
\hline tangens hiperbolni & th & $\mathbb{R}$ & $\frac{e^{x}-e^{-x}}{e^{x}+e^{-x}}$ \\
\hline area sinus hiperbolni & arsh & $\mathbb{R}$ & $\ln \left(x+\sqrt{x^{2}+1}\right)$ \\
\hline area tangens hiperbolni & arth & $\langle-1,1\rangle$ & $\frac{1}{2} \cdot \ln \left(\frac{1-x}{1+x}\right)$ \\
\hline
\end{tabular}

Tablica 1: Pravila pridruživanja i prirodne domene nekih hiperbolnih i area funkcija.

U rješavanju odabranih primjera bit će nam potrebni neki trigonometrijski, odnosno hiperbolni identiteti. Navest ćemo ih u obliku sljedećih lema. Pritom sintagma dopustive vrijednosti znači vrijednosti za koje su definirane lijeva i desna strana jednakosti.

Lema 2. Za sve dopustive vrijednosti $x$ vrijedi:

(a) $\sin (\arcsin x)=\operatorname{tg}(\operatorname{arctg} x)=x$;

(b) $\operatorname{tg}\left(\frac{x}{2}\right)=\frac{1-\cos x}{\sin x}$; 
(c) $\sin x=\frac{\operatorname{tg} x}{\sqrt{1+\operatorname{tg}^{2} x}}$;
(d) $\cos x=\frac{1}{\sqrt{1+\operatorname{tg}^{2} x}}$.

Dokaz. Vidjeti npr. 4].

Lema 3. Za sve dopustive vrijednosti $x$ vrijedi:

(a) $\operatorname{ch}^{2} x-\operatorname{sh}^{2} x=1$;

(b) $\operatorname{th} x=\frac{\operatorname{sh} x}{\operatorname{ch} x}$;

(c) $\operatorname{sh}(\operatorname{arsh} x)=\operatorname{th}(\operatorname{arth} x)=x$

(d) $\operatorname{th}\left(\frac{x}{2}\right)=\frac{\operatorname{sh} x}{1+\operatorname{ch} x}$;

(e) $\operatorname{sh} x=\frac{\operatorname{th} x}{\sqrt{1-\operatorname{th}^{2} x}}$;

(f) $\operatorname{ch} x=\frac{1}{\sqrt{1-\operatorname{th}^{2} x}}$.

Dokaz. Identiteti (a) i (b) slijede izravno iz pravila funkcija ch, sh i th. Podsjetimo da se identitet (a) naziva osnovni hiperbolni identitet.

Identitet (c) slijedi izravno iz definicije inverza funkcije.

(d) Koristeći pravila pridruživanja iz tablice 1 imamo redom:

$$
\begin{aligned}
\operatorname{th}\left(\frac{x}{2}\right) & =\frac{e^{\frac{x}{2}}-e^{-\frac{x}{2}}}{e^{\frac{x}{2}}+e^{-\frac{x}{2}}}=\frac{\left(e^{\frac{x}{2}}-e^{-\frac{x}{2}}\right) \cdot\left(\frac{e^{\frac{x}{2}}+e^{-\frac{x}{2}}}{2}\right)}{\left(e^{\frac{x}{2}}+e^{-\frac{x}{2}}\right) \cdot\left(\frac{e^{\frac{x}{2}}+e^{-\frac{x}{2}}}{2}\right)}=\frac{\frac{e^{x}-e^{-x}}{2}}{\frac{\left(e^{\frac{x}{2}}+e^{-\frac{x}{2}}\right)^{2}}{2}}= \\
& =\frac{\operatorname{sh} x}{\frac{\operatorname{sh} x}{2}}=\frac{\operatorname{sh} x}{\frac{e^{x}+2+e^{-x}}{2}}=\frac{\operatorname{sh}}{1+\operatorname{ch} x} .
\end{aligned}
$$

(e) Transformirat ćemo desnu stranu zadanoga identiteta. Imamo:

$$
\frac{\operatorname{th} x}{\sqrt{1-\operatorname{th}^{2} x}}=\frac{\frac{\operatorname{sh} x}{\operatorname{ch} x}}{\sqrt{1-\frac{\operatorname{sh}^{2} x}{\operatorname{ch}^{2} x}}}=\frac{\frac{\operatorname{sh} x}{\operatorname{ch} x}}{\sqrt{\frac{\operatorname{ch}^{2} x-\operatorname{sh}^{2} x}{\operatorname{ch}^{2} x}}}=\frac{\frac{\operatorname{sh} x}{\operatorname{ch} x}}{\sqrt{\frac{1}{\operatorname{ch}^{2} x}}}=\frac{\frac{\operatorname{sh} x}{\operatorname{ch} x}}{\frac{1}{\operatorname{ch} x}}=\operatorname{sh} x .
$$

(f) Analogno kao (e).

\section{Lema 4.}

$$
\int \frac{d x}{\operatorname{sh} x}=\ln \left|\operatorname{th}\left(\frac{x}{2}\right)\right|+C, C \in \mathbb{R}
$$


Dokaz. Imamo redom:

$$
\begin{aligned}
\int \frac{d x}{\operatorname{sh} x} & =\int \frac{d x}{\frac{e^{x}-e^{-x}}{2}}=\int \frac{2}{e^{x}-e^{-x}} \cdot d x=2 \cdot \int \frac{e^{x}}{e^{2 \cdot x}-1} \cdot d x= \\
& =\left\{\begin{array}{l}
\text { zamjena } \\
t:=e^{x} \\
d t=e^{x} \cdot d x
\end{array}\right\}=2 \cdot \int \frac{1}{t^{2}-1} \cdot d t=\left\{\begin{array}{l}
\text { rastav na } \\
\text { parcijalne } \\
\text { razlomke }
\end{array}\right\} \\
& =2 \cdot \int\left(\frac{1}{2} \cdot\left(\frac{1}{t-1}-\frac{1}{t+1}\right)\right) \cdot d t= \\
& =\int\left(\frac{1}{t-1}-\frac{1}{t+1}\right) \cdot d t=\ln |t-1|-\ln |t+1|= \\
& =\ln \left|\frac{t-1}{t+1}\right|=\ln \left|\frac{e^{x}-1}{e^{x}+1}\right|=\ln \left|\frac{2 \cdot e^{\frac{x}{2}}\left(\frac{e^{\frac{x}{2}}-e^{-\frac{x}{2}}}{2}\right)}{2 \cdot e^{\frac{x}{2}}\left(\frac{e^{\frac{x}{2}}+e^{-\frac{x}{2}}}{2}\right)}\right|= \\
& =\ln \left|\frac{\frac{e^{\frac{x}{2}}-e^{-\frac{x}{2}}}{2}}{\frac{e^{\frac{x}{2}}+e^{-\frac{x}{2}}}{2}}\right|=\ln \left|\frac{\operatorname{sh}\left(\frac{x}{2}\right)}{\operatorname{ch}\left(\frac{x}{2}\right)}\right| \stackrel{\operatorname{lema} 2 \mathrm{~b}}{=} \ln \left|\operatorname{th}\left(\frac{x}{2}\right)\right|+C, C \in \mathbb{R},
\end{aligned}
$$

što smo i željeli pokazati.

Derivacije i neodređeni integrali elementarnih funkcija mogu se naći npr. u [1, 3] i [5], pa ih ovdje ne navodimo.

\section{Primjer 1}

Odredimo standardnu antiderivaciju funkcije $f(x)=\frac{1}{x \cdot \sqrt{1-x^{2}}}$.

\subsection{Rješenje pomoću trigonometrijske zamjene}

Uvedimo zamjenu $t:=\arcsin x$, odnosno $x=\sin t$. Tada je $d x=\cos t \cdot d t$, pa imamo:

$$
\begin{aligned}
\int \frac{1}{x \cdot \sqrt{1-x^{2}}} \cdot d x & =\int \frac{1}{\sin t \cdot \sqrt{1-\sin ^{2} t}} \cdot \cos t \cdot d t= \\
& =\int \frac{\cos t}{\sin t \cdot \sqrt{\cos ^{2} t}} \cdot d t=\int \frac{\cos t}{\sin t \cdot \cos t} \cdot d t= \\
& =\int \frac{d t}{\sin t}=\ln \left|\operatorname{tg}\left(\frac{t}{2}\right)\right|= \\
& =\ln \left|\operatorname{tg}\left(\frac{1}{2} \cdot \arcsin x\right)\right|+C, \quad C \in \mathbb{R} .
\end{aligned}
$$


Odaberemo $C=0$ pa dobijemo: $F(x)=\ln \left|\operatorname{tg}\left(\frac{1}{2} \cdot \arcsin x\right)\right|$.

Većina studenata ovdje će završiti rješavanje zadatka smatrajući da se dobiveno pravilo funkcije $\mathrm{F}$ ne može dalje pojednostavniti. Međutim, u tom je slučaju vrlo teško dokazati da se i hiperbolnom zamjenom dobije jednaka standardna antiderivacija. Zbog toga ćemo dodatno pojednostavniti pravilo funkcije $\mathrm{F}$ primjenom identiteta a) i b) iz leme 1 . Imamo redom:

$$
\begin{aligned}
F(x) & =\ln \left|\operatorname{tg}\left(\frac{1}{2} \cdot \arcsin x\right)\right|=\ln \left|\frac{1-\cos (\arcsin x)}{\sin (\arcsin x)}\right|= \\
& =\ln \left|\frac{1-\sqrt{1-\sin ^{2}(\arcsin x)}}{x}\right|=\ln \left|\frac{1-\sqrt{1-x^{2}}}{x}\right|
\end{aligned}
$$

\subsection{Rješenje pomoću hiperbolne zamjene}

Uvedimo zamjenu $t:=\operatorname{arth} x$, odnosno $x=$ th $t$. Tada je $d x=\frac{1}{\operatorname{ch}^{2} t} \cdot d t$, pa imamo:

$$
\begin{aligned}
\int \frac{1}{x \cdot \sqrt{1-x^{2}}} \cdot d x & =\int \frac{1}{\operatorname{th} t \cdot \sqrt{1-\operatorname{th}^{2} t}} \cdot \frac{1}{\operatorname{ch}^{2} t} \cdot d t= \\
& =\int \frac{1}{\operatorname{th} t} \cdot \frac{1}{\sqrt{1-\operatorname{th}^{2} t}} \cdot \frac{1}{\operatorname{ch}^{2} t} \cdot d t \stackrel{\text { (ema 2b) i 2e) }}{=} \\
& =\int \frac{\operatorname{ch} t}{\operatorname{sh} t} \cdot \operatorname{ch} t \cdot \frac{1}{\operatorname{ch}^{2} t} \cdot d t=\int \frac{d t}{\operatorname{sh} t}= \\
& =\ln \left|\operatorname{th}\left(\frac{t}{2}\right)\right|=\ln \left|\operatorname{th}\left(\frac{1}{2} \cdot \operatorname{arth} x\right)\right|+C, C \in \mathbb{R}
\end{aligned}
$$

Odaberemo $C=0$, pa dobijemo: $F(x)=\ln \left|\operatorname{th}\left(\frac{1}{2} \cdot \operatorname{arth} x\right)\right|$.

I ovdje će većina studenata završiti rješavanje zadatka smatrajući da se dobiveno pravilo funkcije $\mathrm{F}$ ne može više pojednostavniti. Međutim, ipak nije tako. Dobiveno pravilo funkcije F pojednostavnit ćemo koristeći 
sve identitete iz leme 2. Imamo redom:

$$
\begin{aligned}
F(x) & =\ln \left|\operatorname{th}\left(\frac{1}{2} \cdot \operatorname{arth}\right)\right|=\ln \left|\frac{\operatorname{sh}(\operatorname{arth} x)}{1+\operatorname{ch}(\operatorname{arth} x)}\right|= \\
& =\ln \left|\frac{\frac{\operatorname{th}(\operatorname{arth} x)}{\sqrt{1-\operatorname{th}^{2}(\operatorname{arth} x)}}}{1+\frac{1}{\sqrt{1-\operatorname{th}^{2}(\operatorname{arth})}}}\right|=\ln \left|\frac{\frac{x}{\sqrt{1-x^{2}}}}{1+\frac{1}{\sqrt{1-x^{2}}}}\right|=\ln \left|\frac{\frac{x}{\sqrt{1-x^{2}}}}{\frac{\sqrt{1-x^{2}}+1}{\sqrt{1-x^{2}}}}\right|= \\
& =\ln \left|\frac{x \cdot\left(1-\sqrt{1-x^{2}}\right)}{1+\sqrt{1-x^{2}}}\right|=\ln \left|\frac{\left.x+\sqrt{1-x^{2}}\right) \cdot\left(1-\sqrt{1-x^{2}}\right)}{\left(1-\sqrt{1-x^{2}}\right)}\right|=\ln \left|\frac{x \cdot\left(1-\sqrt{\left.1-x^{2}\right)}\right.}{x^{2}}\right|= \\
& =\ln \left|\frac{x \cdot\left(1-\sqrt{1-x^{2}}\right.}{x}\right|, \\
& =\ln \left|\frac{1-\sqrt{1-x^{2}}}{1-}\right|=
\end{aligned}
$$

a to pravilo smo dobili i u točki 2.1 .

\subsection{Usporedba korištenih metoda}

$\mathrm{Na}$ prvi se pogled hiperbolna zamjena čini težom od trigonometrijske. Smatramo da je takva tvrdnja gotovo u cijelosti posljedica nedovoljnoga iskustva u radu s hiperbolnim funkcijama, a ne složenosti same metode. Drugim riječima, metoda trigonometrijske zamjene je preferirana zato što studenti imaju ranije iskustvo u radu s trigonometrijskim funkcijama. Zanemarimo li navedeni problem nedostatka iskustva u radu s hiperbolnim funkcijama, smatramo da metoda hiperbolne zamjene nije lošija za primjenu u odnosu na trigonometrijsku metodu. Smatramo da su potrebne algebarske transformacije približno jednake težine i da su prema tom kriteriju obje metode ravnopravne. Pogledajmo kakva svojstva imaju korišteni identiteti.

Ponajprije, primijetimo da je prirodna domena funkcije $f$ jednaka $D_{f}=\langle-1,1\rangle \backslash\{0\}$. U rješavanju pomoću hiperbolne zamjene taj podatak nam je potreban samo jednom, i to prilikom primjene identiteta $\operatorname{th}(\operatorname{arth} x)=x$ koji vrijedi za svaki $x \in\langle-1,1\rangle$, pa posebno i za svaki $x \in D_{f}$. Svi ostali korišteni (hiperbolni) identiteti vrijede za svaki $x \in \mathbb{R}$. Dakle, prilikom rješavanja pomoću hiperbolne zamjene gotovo da i nije potrebno obraćati pozornost na skup svih dopustivih vrijednosti varijable $x$ za koji vrijede korišteni identiteti.

$\mathrm{S}$ druge strane, smatramo da je jedino za identitet $\sin (\arcsin x)=x$ (relativno) očita njegova valjanost za $x \in D_{f}$. U srednjoj školi studenti su naučili da za svaki $x \in \mathbb{R}$ vrijedi jednakost $\cos x= \pm \sqrt{1-\sin ^{2} x}$, pa 
zapravo nije jasno zašto se prilikom primjene te jednakosti u rješavanju primjera ne koristi znak \pm . Zbog toga je potrebno napomenuti da je $D_{f} \subset\left\langle-\frac{\pi}{2}, \frac{\pi}{2}\right\rangle$ i da je funkcija kosinus strogo pozitivna na $\left\langle-\frac{\pi}{2}, \frac{\pi}{2}\right\rangle$, pa zbog toga za svaki $x \in D_{f}$ vrijede jednakosti $\cos x=\sqrt{1-\sin ^{2} x}$ i $\sqrt{\cos ^{2} t}=\cos t$. Identitet u lemi 1b) vrijedi za $x \in \mathbb{R} \backslash\{k \cdot \pi: k \in \mathbb{Z}\}$, pa je potrebno uočiti i da je $D_{f} \subset \mathbb{R} \backslash\{k \cdot \pi: k \in \mathbb{Z}\}$. Možemo zaključiti da je u ovom slučaju primjereno i potrebno dodatno prokomentirati valjanost korištenih identiteta za svaki $x \in D_{f}$.

Naposljetku, neodređeni integral funkcije $\frac{1}{\sin x}$ je uvršten $\mathrm{u}$ većinu tablica neodređenih integrala elementarnih funkcija (vidjeti npr. tablicu u [1]). On se izvodi korištenjem tzv. univerzalne trigonometrijske zamjene. U dokazu leme 3 vidjeli smo da je neodredeni integral funkcije $\frac{1}{\operatorname{sh} x}$ moguće relativno brzo i jednostavno izvesti na potpuno elementaran način. Zbog toga smatramo da neuvrštavanje neodređenoga integrala funkcije $\frac{1}{\operatorname{sh} x}$ u većinu tablica neodređenih integrala elementarnih funkcija nije značajan nedostatak metode hiperbolne zamjene.

\section{Primjer 2}

Odredimo standardnu antiderivaciju funkcije $g(x)=\frac{1}{x \cdot \sqrt{x^{2}+4}}$.

\subsection{Rješenje pomoću trigonometrijske zamjene}

Uvedimo zamjenu $t:=\operatorname{arctg}\left(\frac{x}{2}\right)$, odnosno $x=2 \cdot \operatorname{tg} t$. Tada je $d x=$ $\frac{2}{\cos ^{2} t} \cdot d t$, pa korištenjem leme $1 \mathrm{~d}$ ) imamo:

$$
\begin{aligned}
\int \frac{1}{x \cdot \sqrt{x^{2}+4}} \cdot d x & =\int \frac{1}{2 \cdot \operatorname{tg} t \cdot \sqrt{4 \cdot \operatorname{tg}^{2} t+4}} \cdot \frac{2}{\cos ^{2} t} \cdot d t= \\
& =\int \frac{\cos t}{\sin t \cdot 2 \cdot \sqrt{\operatorname{tg}^{2} t+1}} \cdot \frac{1}{\cos ^{2} t} \cdot d t= \\
& =\frac{1}{2} \cdot \int \frac{1}{\sin t} \cdot \frac{1}{\sqrt{\operatorname{tg}^{2} t+1}} \cdot \frac{1}{\cos t} \cdot d t= \\
& =\frac{1}{2} \cdot \int \frac{1}{\sin t} \cdot \cos t \cdot \frac{1}{\cos t} \cdot d t= \\
& =\frac{1}{2} \cdot \int \frac{d t}{\sin t}=\frac{1}{2} \cdot \ln \left|\operatorname{tg}\left(\frac{t}{2}\right)\right|= \\
& =\frac{1}{2} \cdot \ln \left|\operatorname{tg}\left(\frac{1}{2} \cdot \operatorname{arctg}\left(\frac{x}{2}\right)\right)\right|+C, C \in \mathbb{R} .
\end{aligned}
$$

Odaberemo $C=0$ pa dobijemo: 


$$
G(x)=\frac{1}{2} \cdot \ln \left|\operatorname{tg}\left(\frac{1}{2} \cdot \operatorname{arctg}\left(\frac{x}{2}\right)\right)\right| .
$$

Primjenom leme 1 pravilo funkcije $G$ dalje transformiramo ovako:

$$
\begin{aligned}
& G(x)=\frac{1}{2} \cdot \ln \left|\operatorname{tg}\left(\frac{1}{2} \cdot \operatorname{arctg}\left(\frac{x}{2}\right)\right)\right|=\frac{1}{2} \cdot \ln \left|\frac{1-\cos \left(\operatorname{arctg}\left(\frac{x}{2}\right)\right)}{\sin \left(\operatorname{arctg}\left(\frac{x}{2}\right)\right)}\right|= \\
& =\frac{1}{2} \cdot \ln \left|\frac{1-\frac{1}{\sqrt{1+\operatorname{tg}^{2}\left(\operatorname{arctg}\left(\frac{x}{2}\right)\right)}}}{\frac{\operatorname{tg}\left(\operatorname{arctg}\left(\frac{x}{2}\right)\right)}{\sqrt{1+\operatorname{tg}^{2}\left(\operatorname{arctg}\left(\frac{x}{2}\right)\right)}}}\right|=\frac{1}{2} \cdot \ln \left|\frac{1-\frac{1}{\sqrt{1+\left(\frac{x}{2}\right)^{2}}}}{\frac{\frac{x}{2}}{\sqrt{1+\left(\frac{x}{2}\right)^{2}}}}\right|= \\
& =\frac{1}{2} \cdot \ln \left|\frac{\frac{\sqrt{1+\left(\frac{x}{2}\right)^{2}}-1}{\sqrt{1+\left(\frac{x}{2}\right)^{2}}}}{\frac{\frac{x}{2}}{\sqrt{1+\left(\frac{x}{2}\right)^{2}}}}\right|=\frac{1}{2} \cdot \ln \left|\frac{\sqrt{1+\left(\frac{x}{2}\right)^{2}}-1}{\frac{x}{2}}\right|= \\
& =\frac{1}{2} \cdot \ln \left|\frac{2 \cdot \sqrt{1+\left(\frac{x}{2}\right)^{2}}-2}{x}\right|=\frac{1}{2} \cdot \ln \left|\frac{\sqrt{x^{2}+4}-2}{x}\right| \text {. }
\end{aligned}
$$

\subsection{Rješenje pomoću hiperbolne zamjene}

Uvedimo zamjenu $t:=\operatorname{arsh}\left(\frac{x}{2}\right)$, odnosno $x=2 \cdot \operatorname{sh} t$. Tada je $d x=$ $2 \cdot \operatorname{ch} t \cdot d t$, pa imamo:

$$
\begin{aligned}
\int \frac{1}{x \cdot \sqrt{x^{2}+4}} \cdot d x & =\int \frac{1}{2 \cdot \operatorname{sh} t \cdot \sqrt{4 \cdot \operatorname{sh}^{2} t+4}} \cdot 2 \cdot \operatorname{ch} t \cdot d t= \\
& =\int \frac{1}{\operatorname{sh} t \cdot 2 \cdot \sqrt{\operatorname{sh}^{2} t+1}} \cdot \operatorname{ch} t \cdot d t \stackrel{\text { lema } 2 \text { a) }}{=} \\
& =\frac{1}{2} \cdot \int \frac{1}{\operatorname{sh} t \cdot \sqrt{\operatorname{ch}^{2} t}} \cdot \operatorname{ch} t \cdot d t= \\
& =\frac{1}{2} \cdot \int \frac{1}{\operatorname{sh} t \cdot \operatorname{ch} t} \cdot \operatorname{ch} t \cdot d t= \\
& =\frac{1}{2} \cdot \int \frac{1}{\operatorname{sh} t} \cdot d t \stackrel{\text { lema }}{=} \frac{1}{2} \cdot \ln \left|\operatorname{th}\left(\frac{t}{2}\right)\right|= \\
& =\frac{1}{2} \cdot \ln \left|\operatorname{th}\left(\frac{1}{2} \cdot \operatorname{arsh}\left(\frac{x}{2}\right)\right)\right|+C, C \in \mathbb{R} .
\end{aligned}
$$

Odaberemo $C=0$, pa dobijemo:

$$
G(x)=\frac{1}{2} \cdot \ln \left|\operatorname{th}\left(\frac{1}{2} \cdot \operatorname{arsh}\left(\frac{x}{2}\right)\right)\right| .
$$


Primjenom svih identiteta iz leme 2 dalje slijedi:

$$
\begin{aligned}
G(x) & =\frac{1}{2} \cdot \ln \left|\operatorname{th}\left(\frac{1}{2} \cdot \operatorname{arsh}\left(\frac{x}{2}\right)\right)\right|=\frac{1}{2} \cdot \ln \left|\frac{\operatorname{sh}\left(\operatorname{arsh}\left(\frac{x}{2}\right)\right)}{1+\operatorname{ch}\left(\operatorname{arsh}\left(\frac{x}{2}\right)\right)}\right|= \\
& =\frac{1}{2} \cdot \ln \left|\frac{\frac{x}{2}}{1+\sqrt{1+\operatorname{sh}^{2}}\left(\operatorname{arsh}\left(\frac{x}{2}\right)\right)}\right|=\ln \left|\frac{x}{2 \cdot\left(1+\sqrt{1+\left(\frac{x}{2}\right)^{2}}\right)}\right|= \\
& =\ln \left|\frac{x}{2+\sqrt{4 \cdot\left(1+\left(\frac{x}{2}\right)^{2}\right)}}\right|=\ln \left|\frac{x}{2+\sqrt{4+x^{2}}}\right|= \\
& =\ln \left|\frac{x \cdot\left(\sqrt{x^{2}+4}-2\right)}{\left(2+\sqrt{4+x^{2}}\right) \cdot\left(\sqrt{x^{2}+4}-2\right)}\right|=\ln \left|\frac{x \cdot\left(\sqrt{x^{2}+4}-2\right)}{\left(x^{2}+4\right)-4}\right|= \\
& =\ln \left|\frac{x \cdot\left(\sqrt{x^{2}+4}-2\right)}{x^{2}}\right|=\ln \left|\frac{\sqrt{x^{2}+4}-2}{x}\right|,
\end{aligned}
$$

što smo i željeli pokazati.

\subsection{Usporedba korištenih metoda}

U ovome je slučaju prednost korištenja metode hiperbolne zamjene još izraženija nego u primjeru 1. Naime, primijetimo da je prirodna domena funkcije $g$ jednaka $D_{g}=\mathbb{R} \backslash\{0\}$. Korišteni hiperbolni identiteti vrijede za svaki $x \in \mathbb{R}$, pa posebno i za svaki $x \in D_{g}$. Dakle, nije potrebno provesti nikakve dodatne analize, komentare i sl. vezane uz korištene identitete.

Međutim, korišteni identiteti iz leme 1 ovdje zahtijevaju dodatnu analizu. Naime, jedino je identitet $\operatorname{tg}(\operatorname{arctg} x)=x$ istinit za svaki $x \in \mathbb{R}$, pa posebno i za svaki $x \in D_{g}$. Znači li to da ostale identitete zapravo nismo smjeli primijeniti, odnosno da smo trebali dodati neke uvjete uz koje bismo ih mogli primijeniti?

Odgovor na ovo pitanje je negativan. Naime, u rješenju primjera 2 navedenom u točki 3.1. se kao argument funkcija sin, cos i tg pojavljuje funkcija $g_{1}(x)=\operatorname{arctg}\left(\frac{x}{2}\right)$. Ona ima prirodnu domenu $\mathbb{R}$ i sliku $\left\langle-\frac{\pi}{2}, \frac{\pi}{2}\right\rangle$. $\mathrm{U}$ točki 2.3. smo istaknuli da je na tom intervalu funkcija kosinus strogo pozitivna. Zbog toga za svaki $x \in \mathbb{R}$, a time i za svaki $x \in D_{g}$, vrijedi identitet

$$
\cos (\operatorname{arctg} x)=\frac{1}{\sqrt{1+\operatorname{tg}^{2}(\operatorname{arctg} x)}}=\frac{1}{\sqrt{1+x^{2}}}
$$


Iz istoga razloga (stroga pozitivnost kosinusa) predznak funkcije tg na $\left\langle-\frac{\pi}{2}, \frac{\pi}{2}\right\rangle$ jednak je predznaku funkcije sin. Odatle slijedi da za svaki $x \in \mathbb{R}$, a time i za svaki $x \in D_{g}$, vrijedi identitet:

$$
\sin (\operatorname{arctg} x)=\frac{\operatorname{tg}(\operatorname{arctg} x)}{\sqrt{1+\operatorname{tg}^{2}(\operatorname{arctg} x)}}=\frac{x}{\sqrt{1+x^{2}}} .
$$

Dakle, ni $\mathrm{u}$ jednom od navedenih identiteta ispred znaka drugoga korijena nije potreban znak \pm .

Zaključno, promotrimo identitet

$$
\operatorname{tg}\left(\frac{1}{2} \cdot \operatorname{arctg}\left(\frac{x}{2}\right)\right)=\frac{1-\cos \left(\operatorname{arctg}\left(\frac{x}{2}\right)\right)}{\sin \left(\operatorname{arctg}\left(\frac{x}{2}\right)\right)} .
$$

Njegova lijeva strana je definirana za sve $x \in \mathbb{R}$, dok desna strana nije definirana samo za $x=0$. Zbog toga taj identitet vrijedi za svaki $x \in \mathbb{R} \backslash\{0\}=D_{g}$. Time smo obrazložili da svi korišteni identiteti vrijede na $D_{g}$.

Metoda trigonometrijske zamjene u ovom slučaju zahtijeva dodatnu netrivijalnu analizu korektnosti postupka rješavanja. Zbog nedostatka raspoloživoga vremena na nastavi, ta analiza se uglavnom izostavlja ili se tek uzgredno napomene da korišteni identiteti vrijede za svaki $x \in D_{g}$. Na temelju vlastitoga iskustva uvjereni smo da je relativno mali broj studenata stručnih studija sposoban ispravno obrazložiti valjanost primjene tih identiteta i da spomenutu analizu zato ne bi trebalo izostavljati.

\section{Zaključak}

U nastavi matematičkih predmeta na veleučilištima, samostalnim visokim školama i fakultetima, a posebno na onima koji izvode tehničke sveučilišne i stručne studije, rješavaju se i različiti zadaci iz integralnoga računa. U te zadatke pripadaju i posebni tipovi integrala iracionalnih funkcija. Neki od tih tipova mogu se rješavati koristeći metodu trigonometrijske zamjene i metodu hiperbolne zamjene. Zbog iskustva studenata $\mathrm{u}$ radu s trigonometrijskim funkcijama, kao i njihova nedovoljnoga iskustva u radu s hiperbolnim funkcijama, u rješavanju se najčešće koristi metoda trigonometrijskih zamjena. U ovom radu smo nastojali pokazati da metoda hiperbolnih zamjena u takvim slučajevima nije lošija od metode trigonometrijskih zamjena, a u nekim slučajevima je jednostavnija jer ne zahtijeva dodatne analize korektnosti provedena postupka. Nadamo se da će se u budućnosti stvoriti potrebni preduvjeti da se u okviru nastavnih programa matematičkih predmeta mogu detaljnije obraditi hiperbolne funkcije i njihove primjene u rješavanju različitih praktičnih problema. 


\section{Literatura}

[1] B. P. Demidovič, Zadaci i riješeni primjeri iz matematičke analize za tehničke fakultete, Golden marketing, Zagreb, 2003.

[2] B. Kovačić, L. Marohnić, T. Strmečki, Repetitorij matematike za studente elektrotehnike, Golden marketing, Tehničko veleučilište, Zagreb, 2016.

[3] S. Kurepa, Matematička analiza 1, Diferenciranje i integriranje, Školska knjiga, Zagreb, 1997.

[4] B. Pavković, D. Veljan, Elementarna matematika 2, Školska knjiga, Zagreb, 1995.

[5] I. Slapničar, Matematika 2, Fakultet elektrotehnike, strojarstva i brodogradnje Sveučilišta u Splitu, Split, 2008.

Denis Benčec

student 2. godine preddiplomskoga stručnoga studija elektrotehnike,

Tehničko veleučilište u Zagrebu Elektrotehnički odjel, Konavoska 2, Zagreb

E-mail adresa: denis.bencec@tvz.hr

Bojan Kovačić

Tehničko veleučilište u Zagrebu Elektrotehnički odjel, Konavoska 2, Zagreb

E-mail adresa: bojan.kovacic@tvz.hr 